\title{
(2) OPEN ACCESS \\ Switching among branded and generic medication products during ongoing treatment of psychiatric illness
}

\author{
Jeffrey Habert (D) , ${ }^{1}$ Howard C Margolese, ${ }^{2}$ Adriana Wilson, ${ }^{3}$ \\ Matthieu Boucher, ${ }^{4,5}$ Pierre Blier $^{6}$
}

${ }^{1}$ Department of Family and Community Medicine, University of Toronto, Thornhill, Ontario, Canada

2Department of Psychiatry, McGill University, Montreal, Québec, Canada

${ }^{3}$ Department of Psychiatry, Dalhousie University, Halifax, Nova Scotia, Canada ${ }^{4}$ Pfizer Canada Inc, Montréal, Kirkland, Canada

${ }^{5}$ Department of Pharmacology and Therapeutics, McGill University, Montréal, Québec, Canada

${ }^{6}$ Departments of Psychiatry and Cellular \& Molecular Medicine, University of Ottawa Institute of Mental Health Research, Ottawa, Ontario, Canada

\section{Correspondence to}

Dr Jeffrey Habert, University of Toronto, Toronto, ON M5S, Canada; jhabert@rogers.com

Received 27 June 2019 Revised 11 September 2019 Accepted 13 December 2019 Published Online First 10 January 2020

\begin{abstract}
Switches between branded (reference) medications and the corresponding generic medications or between two different corresponding generic medications occur commonly during the treatment of central nervous system disorders. Prescribing a generic product in place of a reference product can reduce patient and pharmacy costs. But there can be implications. Planned or unplanned switches from one product to another during ongoing treatment may introduce variability in drug exposure which could in turn compromise efficacy and/or tolerability. Studies comparing the initiation of reference versus generic products do not provide clear evidence of the superiority of reference or generic products generally, whereas several studies examining a switch between reference and generic products suggest that reductions in efficacy or medication adherence and persistence may be associated with generic substitution. Clinicians should work with patients to facilitate a consistent supply of reference or generic drug product that provides stable exposure to avoid clinical deterioration or poor tolerability.
\end{abstract}

\section{INTRODUCTION}

The development of drugs with novel mechanisms of action or those directed to new therapeutic targets are associated with very high costs, which are reflected in drug pricing. ${ }^{1}$ Once patent or data protection for a branded product (reference) has expired, generic versions, with fewer approval requirements and lower associated development costs enter the market. ${ }^{2}$ Generic products with central nervous system (CNS) indications may provide the most substantial cost savings, given that over $90 \%$ of novel CNS drugs in clinical trials fail to receive approval, even after significant investment. ${ }^{3}$ Patients receiving medication for psychiatric illness are commonly switched from a reference product to a generic version at the pharmacy when a generic product becomes available. ${ }^{4}$ Prescribers understand that switches to generics may occur unless they specifically indicate 'dispense as written' or 'no substitution'. 6 In many circumstances, switches between different generic versions of the reference drug (unplanned switches) at the pharmacy level may be made without the knowledge of the patient or the clinician.

A generic product is required to be pharmaceutically equivalent to (ie, having the same active ingredient or ingredients, the same dosage form and route of administration, and identical strength) and to demonstrate bioequivalence with (ie, having the same rate and extent of absorption of active drug, within 80\%-125\% bioavailability) the reference product. $^{78}$ Efficacy and safety data are not required for the approval of a new generic product. Agencies tasked with generic drug approval expect that generic products demonstrating bioequivalence with their reference product will have no clinically important efficacy or safety differences from the reference. 910 Nonetheless, a substantial proportion of patients and physicians queried in one study thought that generic products may be less safe and effective compared with the reference product, ${ }^{11}$ reflecting the larger question of whether bioequivalence reliably translates to therapeutic equivalence, $^{12}$ and more specifically, whether 


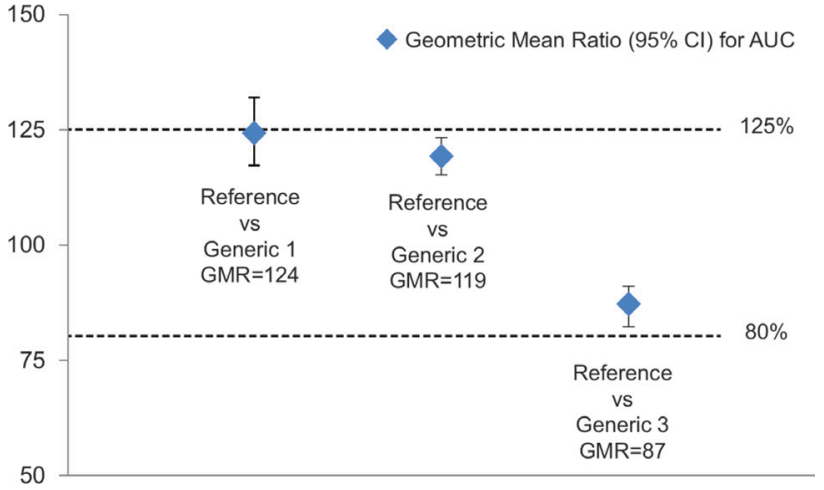

Figure 1 Hypothetical bioequivalence tests illustrating possible relationships between a reference product and several generic products. Generic product one fails to meet the bioequivalence criteria with the reference drug: the $90 \%$ $\mathrm{Cl}$ of the geometric mean ratio for AUC falls outside the $80 \%-125 \%$ bioequivalence limits. Generic products 2 and 3 demonstrate bioequivalence with the reference drug. However, a bioequivalence test comparing generic products 2 and 3 might fail, a circumstance that, although rare, might affect safety or efficacy in the event of a switch from one to the other. $A \cup C$, area under the plasma concentration-time curve; GMR, geometric mean ratio.

the efficacy and safety of a psychotropic medication will remain consistent through planned or unplanned switches between reference and generic products or between different generic products.

\section{HOW MUCH VARIABILITY IS THERE BETWEEN PRODUCTS WITH DEMONSTRATED BIOEQUIVALENCE?}

The USA, Canada and the European Union provide similar (but not identical) criteria for demonstrating bioequivalence between two products. ${ }^{9} 1314$ The criteria impose limits on the variability between generic and reference products in peak plasma concentration $\left(\mathrm{C}_{\mathrm{max}}\right)$ and overall drug exposure (measured as area under the plasma concentration-time curve (AUC)). For example, the Canadian criteria state that the upper and lower limits of the $90 \%$ CI for the ratio of geometric mean AUC for test (in this case, generic) and reference products should fall within a $80 \%-125 \%$ bioequivalence limit. ${ }^{9}$ The ratio of exposure for reference versus generic falls within a smaller range; that is, if exposure for the reference and generic products differs by as much as $25 \%$, the upper confidence limit would fall outside the bioequivalence range, and the bioequivalence test would fail (figure 1). ${ }^{15}$

Additional variability can, however, be seen between two products that have demonstrated bioequivalence. Products that provide similar exposure under the specific conditions of a pharmacokinetics study may diverge under other circumstances. First, bioequivalence studies typically enrol a small number of healthy volunteers, often young, male and Caucasian adults with no chronic medical issues, who are not representative of the patients who will use the medication. ${ }^{16}{ }^{17}$ In addition, products that demonstrate bioequivalence after single-dose administration may have peak plasma concentrations that fall outside bioequivalence criteria under steady-state conditions, ${ }^{18}$ or products that are bioequivalent in a fasting state could provide different exposures under fed conditions. ${ }^{19}$ For some drugs, batch-to-batch variability in product pharmacokinetics might be great enough that bio-inequivalence could occur between batches of the same product, ${ }^{20}$ potentially due to differences in excipients such as fillers, colourings or coatings. In addition, there is likely a cumulative effect of variability throughout the lifecycle of both generic and reference products as batch sizes are increased, manufacturing sites are added or changed, and additional formulations are produced. ${ }^{12}{ }^{15}$ Finally, two generic products may differ even more from one another than they do from the reference product: one generic product might be associated with lower exposure compared with the reference and the second with higher exposure (figure 1), and as variability increases over the reference product's lifecycle, differences between successive generic products may grow with increasing time between their introduction. Overall, the likelihood of clinically important differences between a generic product and its reference has been shown to be very low: in a survey of 2070 bioequivalence studies of generic solid dosage form products versus their references over a 12-year period, the average of geometric mean ratios for both $\mathrm{C}_{\max }$ and AUC was 1.00, with an average difference $<5 \%$ for both measures. ${ }^{21}$ In contrast, a smaller survey of 97 bioequivalence studies for modified release formulations of antiepileptic drugs, reported that $\mathrm{C}_{\max }$ varied by $>15 \%$ in $26 \%$ of studies. ${ }^{22}$ Neither survey included comparisons between generic products. It is possible, therefore, for switches, particularly from one generic product to another, to result in clinically significant increases or decreases in plasma drug levels, with potential effects on efficacy or safety. ${ }^{12}$ In a single study that assessed bioequivalence among four generic products with the same reference, using adjusted indirect comparisons, each pair demonstrated bioequivalence based on AUC but exceeded the acceptance range in 2 of 6 comparisons for $\mathrm{C}_{\max }{ }^{23} \mathrm{~A}$ large-scale survey comparing generic products with each other is warranted.

\section{OUTCOMES AFTER PSYCHOTROPIC MEDICATION SWITCH}

Studies comparing outcomes for reference versus generic products have generally asked one of three questions: (1) Are generic products therapeutically equivalent (within 80\%-125\% bioequivalence) to their reference counterparts? (2) Does switching from reference to generic products negatively affect patient outcomes? or (3) Does any switch (ie, from reference to generic, from generic to reference or from generic 
Table 1 Evidence of effects of switching medication products during the treatment of psychiatric illness; based on studies published in 2010-2017 of medications for seizures or bipolar disorder, major depressive disorder, psychosis and attention deficit disorder. Reviewed in Blier, $2018^{12}$

\begin{tabular}{ll}
\hline Study type (number of studies) & Findings \\
\hline Initiation of reference versus generic: & Are generic products therapeutically equivalent to their reference counterparts? \\
Retrospective database analyses (five studies) & - Lower healthcare resource use for generic products in some studies, for reference in others \\
& -
\end{tabular}

to generic) affect patient outcomes? Findings from a recent review by Blier et al of studies of psychotropic medications that addressed these questions are summarised in table $1 .^{12}$

The first question has been addressed by comparing healthcare resource use, medication persistence, prescribing changes and efficacy outcomes for patients initiated with reference versus generic products based on retrospective reviews of medical record and claims database data. Results from those analyses are mixed, with some studies favouring the reference product and others favouring the generic product. The variation in study outcomes is consistent with the understanding that generic products may be associated with higher or lower exposures compared with the reference product, and while lower exposure can reduce drug efficacy, higher exposure might compromise tolerability. The studies suggest that, on average, generic products generally may be therapeutically equivalent to their reference counterparts at initiation of treatment. ${ }^{12}$

The majority of studies examined in the Blier et al review addressed the question of whether switching from a reference product to a generic product results in negative patient outcomes. Studies that addressed this question included prospective trials and retrospective database studies, each comparing either preswitch versus postswitch time periods or patients who switched to a generic compared with those who continued taking the reference product. ${ }^{12}$ Although no significant effects of a reference-to-generic switch were observed in some of the reviewed studies, the switch to a generic product was associated with reduced treatment persistence, increased dose or medication changes, decreased treatment satisfaction and evidence of clinical deterioration in some of the studies. ${ }^{12}$ Given that generic products did not consistently perform more poorly than their reference product in studies that compared outcomes for patients initiated on reference versus generic products, it is likely that in these switching studies, the switch itself contributes to negative outcomes.

It is critical to ask, then, whether any type of switch between products-reference to generic, generic to reference or generic to generic-can negatively affect patient outcomes compared with continuing treatment on a single product, either reference or generic. Few studies have addressed this issue, and none have examined switches from one generic product to another. ${ }^{12}$ To date, evidence suggests that when a switch between products is associated with poorer outcomes, it is the lack of continuity on a single product, rather than the specific product to which the patient is switched, that is problematic. ${ }^{12}$ Additional studies are needed to confirm this conclusion. It should also be noted that changes in a product unrelated to bioequivalence, such as the taste or appearance of the product, can affect patient behaviour, such as adherence, which can, in turn, lead to poor treatment outcomes. ${ }^{24}$

\section{CONCLUSIONS}

There are immediate economic incentives in terms of medication cost for switching patients between 


\section{Box 1 Key points for clinicians}

- Reference and generic products are not identical. Although the API is the same, small differences in constitution can affect efficacy or safety in individual patients.

- Modified release products are complex; the delivery systems are difficult to reproduce. Some generics do not release the $A P I(s)$ at the same rate.

- Avoid unnecessary or unplanned product switches (reference product-to-generic, generic-to-generic or even generic-to-reference product); note on the prescription: 'Use SAME BRAND', 'Do not change product' or 'SAME PRODUCT'.

- Communicate directly with pharmacists to be sure they recognise that reference and generic products may differ.

- Consider whether an unplanned product switch may be a factor when any sudden deterioration in symptomatology is observed.

- Ensure that the therapeutic team is aware of any switch so that the patient can be switched back if there are negative consequences.

- Clinicians should make sure that patients are aware that switches between products can occur with new prescriptions; in the case of a switch, changes in shape or colour of the medication might occur.

API, active pharmaceutical ingredient.

branded and generic products during the course of their treatment. While studies have demonstrated that generic products are generally therapeutically equivalent to reference products, ${ }^{12}$ product discontinuity resulting from unplanned switches during treatment with psychotropic medication may be associated with negative patient outcomes and/or higher longer-term healthcare costs. Evidence suggests that switches between reference or generic products have the potential to reduce efficacy and/or increase adverse event burden. Generic-to-generic switches may be most problematic, as the variability in drug exposure may be greater. In addition, generic-to-generic switches are generally unplanned and neither the patient nor the clinician is aware of the medication change. There is thus a critical unmet need to conduct studies that examine the impact of switching between generic products during the ongoing treatment of patients with psychotropic medications. Until then, clinicians should advocate for the patient and work to facilitate a consistent and continued supply of the drug product (whether reference or generic) that provides stability and avoids clinical deterioration as a key treatment goal (box 1). Unplanned changes in the treatment plan, even one considered minimal such as a medication switch, could have detrimental effects in some patients.

Acknowledgements Medical writing support was provided by Kathleen M Dorries, PhD, of Peloton Advantage, LLC, an OPEN Health company, and was funded by Pfizer Canada.
Contributors Manuscript preparation: JH, $\mathrm{HCM}, \mathrm{AW}, \mathrm{MB}$ and $\mathrm{PB}$; Manuscript review and revisions: JH, HCM, AW, MB and PB; Final approval of manuscript: JH, HCM, AW, MB and PB.

Funding The authors have not declared a specific grant for this research from any funding agency in the public, commercial or not-for-profit sectors.

Competing interests $\mathrm{JH}$ : Honoraria/advisory board/speaker: Pfizer, Amgen, BMS, Bayer, Boehringer, Eli-Lilly, Purdue, Allergan, AstraZeneca, Lundbeck, Novo-Nordisk, Servier, Janssen, Aralez. HCM: Honoraria/paid speaker: BMS, Janssen, Lundbeck, Otsuka, Sunovion; Research support: Acadia, Lundbeck, Janssen; Consultant: HLS Therapeutics, Janssen, Lundbeck, Otsuka, Perdue, Pfizer, Shire, Sunovion; No stocks or employment. AW: Consultant for Pfizer. MB: Former employee of Pfizer Canada Inc at the time of writing this manuscript. PB: Honoraria for advisory board participation, consultancy, grant funding, and/or giving lectures for Allergan, BMS, Janssen, Lundbeck, Otsuka, Pfizer, Sunovion, Takeda.

Patient consent for publication Not required.

Provenance and peer review Not commissioned; externally peer reviewed.

Data availability statement There are no data in this work.

Open access This is an open access article distributed in accordance with the Creative Commons Attribution Non Commercial (CC BYNC 4.0) license, which permits others to distribute, remix, adapt, build upon this work non-commercially, and license their derivative works on different terms, provided the original work is properly cited, appropriate credit is given, any changes made indicated, and the use is non-commercial. See: http://creativecommons.org/ licenses/by-nc/4.0/.

\section{ORCID iD}

Jeffrey Habert http://orcid.org/0000-0003-0009-3182

\section{REFERENCES}

1 DiMasi JA, Grabowski HG, Hansen RW. Innovation in the pharmaceutical industry: New estimates of R\&D costs. $J$ Health Econ 2016;47:20-33.

2 Borgheini G. The bioequivalence and therapeutic efficacy of generic versus brand-name psychoactive drugs. Clin Ther 2003;25:1578-92.

3 Riordan HJ, Cutler NR. The death of CNS drug development: overstatement or omen? J Clin Studies 2012;3:12-15.

4 Murawiec S, Rajewska-Rager A, Samochowiec J, et al. Pharmacy switch of antipsychotic medications: patient's perspective. Ann Gen Psychiatry 2015;14:31.

5 Lessing C, Ashton T, Davis P. Do users of risperidone who switch brands because of generic reference pricing fare better or worse than non-switchers? a new Zealand natural experiment. Adm Policy Ment Health 2015;42:695-703.

6 Competition Bureau Canada. Canadian generic drug sector study. Gatineau, Quebec, Canada: Competition Bureau Canada, 2007.

7 Health Canada. Guidance document: conduct and analysis of comparative bioavailability studies, 2012. Available: https:// www.canada.ca/en/health-canada/services/drugs-healthproducts/drug-products/applications-submissions/guidancedocuments/bioavailability-bioequivalence/conduct-analysiscomparative.html\#a [Accessed 18 Sep 2017].

8 Seoane-Vazquez E, Rodriguez-Monguio R, Hansen R. Interchangeability, safety and efficacy of modified-release drug formulations in the USA: the case of opioid and other nervous system drugs. Clin Drug Investig 2016;36:281-92.

9 Health Canada. Guidance document: comparative bioavailability standards: formulations used for systemic effects, 2012. Available: https://www.canada.ca/en/health- 
canada/services/drugs-health-products/drug-products/ applications-submissions/guidance-documents/bioavailabilitybioequivalence/comparative-bioavailability-standardsformulations-used-systemic-effects.html [Accessed 10 Aug 2018].

10 Drugs@FDA Glossary of Terms, 2017. Available: https://www. fda.gov/drugs/informationondrugs/ucm079436.htm [Accessed 5 Nov 2018].

11 Colgan S, Faasse K, Martin LR, et al. Perceptions of generic medication in the general population, doctors and pharmacists: a systematic review. BMJ Open 2015;5:e008915.

12 Blier P, Margolese HC, Wilson EA, et al. Switching medication products during the treatment of psychiatric illness. Int $J$ Psychiatry Clin Pract 2019;23:2-13.

13 Guidance for Industry. Statistical approaches to establishing bioequivalence, 2001. Available: https://www.fda.gov/ downloads/drugs/guidances/ucm070244.pdf [Accessed November 5, 2018].

14 European Medicines Agency. Guideline on the investigation of bioequivalence, 2010. Available: http://www.ema.europa.eu/ docs/en_GB/document_library/Scientific_guideline/2010/01/ WC500070039.pdf [Accessed 18 Jan 2018].

15 Meyer MC. United States food and drug administration requirements for approval of generic drug products. J Clin Psychiatry 2001;62:4-9. Discussion 23-4.

16 Food and Drug Administration. Guidance for industry: bioavailability and bioequivalence studies for orally administered drug products - general considerations, revision 1 2003.
17 Health Canada. The safety and effectiveness of generic drugs, 2012. Available: https://www.canada.ca/content/dam/hc-sc/ migration/hc-sc/hl-vs/alt_formats/pdf/iyh-vsv/med/med-geneng.pdf [Accessed 23 Dec 2019].

18 Tothfalusi L, Endrenyi L. Approvable generic carbamazepine formulations may not be bioequivalent in target patient populations. Int J Clin Pharmacol Ther 2013;51:525-8.

19 Waldman SA, Morganroth J. Effects of food on the bioequivalence of different verapamil sustained-release formulations. J Clin Pharmacol 1995;35:163-9.

20 Burmeister Getz E, Carroll KJ, Mielke J, et al. Betweenbatch pharmacokinetic variability inflates type I error rate in conventional bioequivalence trials: a randomized advair diskus clinical trial. Clin Pharmacol Ther 2017;101:331-40.

21 Davit BM, Nwakama PE, Buehler GJ, et al. Comparing generic and innovator drugs: a review of 12 years of bioequivalence data from the United States food and drug administration. Ann Pharmacother 2009;43:1583-97.

22 Johnson EL, Chang Y-T, Davit B, et al. Assessing bioequivalence of generic modified-release antiepileptic drugs. Neurology 2016;86:1597-604.

23 Pejčić Z, Vučićević K, García-Arieta A, et al. Adjusted indirect comparisons to assess bioequivalence between generic clopidogrel products in Serbia. Br J Clin Pharmacol 2019;85:2059-65.

24 Kesselheim AS, Misono AS, Shrank WH, et al. Variations in pill appearance of antiepileptic drugs and the risk of nonadherence. JAMA Intern Med 2013;173:202-8. 